УДК 513.88

\title{
Algebraic Analysis of Differential Equations
}

\author{
Tatyana A.Osetrova* \\ Institute of Mathematics \\ Siberian Federal University \\ Svobodny 79, Krasnoyarsk, 660041 \\ Russia \\ Nikolai Tarkhanov ${ }^{\dagger}$ \\ Institut für Mathematik \\ Universität Potsdam \\ Am Neuen Palais 10, 14469 Potsdam \\ Germany
}

Received 10.07.2008, received in revised form 20.09.2008, accepted 05.10.2008

Given any algebra over a field with a finite number of generators, we define a first order partial differential operator acting on functions taking their values in the algebra. While being not canonical, the construction is fairly natural. We call this differential operator Dirac operator related to the algebra, and show some examples. Conversely, to each homogeneous first order differential operator one assigns an algebra which absorbs formal properties of the operator.

Keywords: normed algebras.

\section{Introduction}

In modern mathematics there are number systems with complex units, more precisely complex numbers, quaternions, Cayley numbers, etc. By a number system is meant any division algebra with unity. In these systems one can fix 1,3 or 7 elements $g_{j}$, respectively, which along with unity span the number system as real vector space.

Sometimes this does not suffice. For an arbitrary integer $n>0$ one looks for structures which contain both the real numbers and elements $g_{1}, \ldots, g_{n}$ and where a product is defined to satisfy certain conditions. The elements $g_{1}, \ldots, g_{n}$ are called generators of the algebra.

The most remarkable structures of such a type are the Clifford and Graßmann algebras. The former algebra discovered by W.K. Clifford (1878) is of great importance in differential geometry and quantum physics. The latter discovered by H. Graßmann (1844) has moreover found impressive applications in partial differential equations.

Any advanced theory of functions with values in an algebra includes a basic differential equation whose solutions fit well to the algebraic structure. Examples are given by holomorphic functions of a complex variable which satisfy the Cauchy-Riemann equation, or quaternionic functions satisfying the W. Hamilton equation (1843).

The main idea of this paper is quite classical and goes as far as to Dirac (1928) who wanted to quantise the electron. As usual by a Dirac operator is meant any matrix factorisation of the Laplace operator. In this sense all the operators mentioned above are Dirac operators, and we continue to use this designation for abstract algebras.

\footnotetext{
*e-mail: osetrova@lan.krasu.ru

†e-mail: tarkhanov@math.uni-potsdam.de

(c) Siberian Federal University. All rights reserved
} 


\section{Dirac operator}

Through this work, by an algebra $\mathcal{A}$ we mean an algebra over a fixed ground field $\mathbb{K}$. Thus, $\mathcal{A}$ is a vector space over $\mathbb{K}$ along with a $\mathbb{K}$-bilinear mapping $\mathcal{A} \times \mathcal{A} \rightarrow \mathcal{A}$ which is called multiplication and denoted by $a \cdot b$ or simply $a b$, if it does not cause any confusion. The properties "associative," "commutative" or "unital" are often required without any explicit explanation.

Example 1. A familiar example is the cross product in $\mathbb{R}^{3}$. This is even a Lie algebra.

The dimension of $\mathcal{A}$ as a $\mathbb{K}$-vector space is called the rank of the algebra $\mathcal{A}$. By definition, any algebra $\mathcal{A}$ of a finite rank $r$ over $\mathbb{K}$ possesses a basis $e_{1}, \ldots, e_{r}$, and multiplication in the algebra is completely determined by multiplication of the basis elements. Since every product $e_{j} e_{k}$ is again an element of $\mathcal{A}$, it can be written in the form

$$
e_{j} e_{k}=\sum_{i=1}^{r} a_{j, k}^{i} e_{i}
$$

for $j, k=1, \ldots, r$.

The scalars $a_{j, k}^{i}$ are called structure constants of the algebra. They define multiplication by

$$
\left(\sum_{j=1}^{r} y^{j} e_{j}\right)\left(\sum_{k=1}^{r} z^{k} e_{k}\right)=\sum_{i=1}^{r}\left(\sum_{\substack{j=1, \ldots, r \\ k=1, \ldots, r}} a_{j, k}^{i} y^{j} z^{k}\right) e_{i} .
$$

Relations (1) give a multiplication table for the algebra $\mathcal{A}$. Evidently, the structure constants can not be given arbitrarily, for they have to guarantee the associativity law and (if required) the existence of unity.

Any function $y$ on an open set $\mathcal{O} \subset \mathbb{R}^{r}$ with values in $\mathcal{A}$ can be written as

$$
y(x)=\sum_{j=1}^{r} y^{j}(x) e_{j},
$$

with $y^{j}$ a function on $\mathcal{O}$ with values in $\mathbb{K}$. In this way $y$ can be specified within functions on $\mathcal{O}$ taking their values in $\mathbb{K}^{r}$.

The expression

$$
D=\sum_{j=1}^{r} e_{j} \frac{\partial}{\partial x^{j}}
$$

is called the Dirac operator for the algebra $\mathcal{A}$. It acts naturally on $\mathcal{A}$-valued functions by

$$
D y=\sum_{j, k=1}^{r}\left(\frac{\partial}{\partial x^{j}} y^{k}\right) e_{j} e_{k}=\sum_{i=1}^{r} \sum_{k=1}^{r}\left(\sum_{j=1}^{r} a_{j, k}^{i} \frac{\partial}{\partial x^{j}}\right) y^{k} e_{i} .
$$

When thinking of $y$ as an $r$-column of $\mathbb{K}$-valued functions, we arrive at a matrix representation of $D$,

$$
A=\left(\sum_{j=1}^{r} a_{j, k}^{i} \frac{\partial}{\partial x^{j}}\right)_{\substack{i=1, \ldots, r \\ k=1, \ldots, r}} .
$$

The system $A y=0$ obtained this way has several peculiarities. Firstly, it is of order 1 . Secondly, the number of equations is equal to the number of unknown functions and this just 
amounts to the number of independent variables. And thirdly, the differential operator $A$ has constant coefficients and is homogeneous. Hence, when trying to represent an arbitrary partial differential operator $A$ of order $\geqslant 2$ which maps functions on an open set $\mathcal{O} \subset \mathbb{R}^{n}$ with values in $\mathbb{K}^{k}$ to functions on $\mathcal{O}$ with values in $\mathbb{K}^{\ell}$ in the form (2), we need to get rid of these restrictions. Firstly, any system of partial differential equations of high order can be reduced in a familiar way to a first order system. Secondly, one can assume without loss of generality that the number of equations $\ell$ is greater than or equal to both $k$ and $n$, for if not, we add a number of trivial equations to the system. Set $r=\ell$. Then $k<r$ corresponds to the case when $y^{k+1}, \ldots, y^{r}$ are all zero, and $n<r$ corresponds to the case when the unknown function $y$ is independent of $x^{n+1}, \ldots, x^{r}$. This means in practice that suitable structural constants $a_{j, k}^{i}$ vanish. And thirdly, in the case of variable coefficients we have to deal with an algebra bundle over $\mathcal{O}$ rather than with a single algebra. Moreover, a proper choice of the matrix $T(x)$ in the equation $A(T y)=0$ enables one in many cases to transform $A y=0$ into a homogeneous system.

We now start with a given matrix of partial differential operators of the form (3). Let $\mathcal{A}$ be an $r$-dimensional vector space over $\mathbb{K}$ with the basis $\left\{e_{1}, \ldots, e_{r}\right\}$. We give $\mathcal{A}$ the algebra structure by (1) where $a_{j, k}^{i}$ are defined in (3). Regarding $\mathbb{K}^{r}$-valued functions on $\mathbb{R}^{r}$ as functions with values in $\mathcal{A}$, we introduce the differential operator $(2)$. Then the analysis above shows that the matrix representation of this operator is exactly (3). We have thus proved

Theorem 1. For any partial differential operator $A$ with constant coefficients on $\mathbb{R}^{n}$ there is a finite-dimensional algebra $\mathcal{A}$ over $\mathbb{K}$, such that $A$ has the form (2).

The advantage of this approach lies in the fact that $\mathcal{A}$ actually absorbs the algebraic properties of $A$. The study of $\mathcal{A}$ might highlight particular symmetry properties of the space of solutions to $A y=0$.

\section{Example for Non-Associativity}

For general first order partial differential operators the algebra $\mathcal{A}$ need not be good. Not only is it non-commutative or non-unital but also fails to be associative in general.

Example 2. Consider the equation

$$
\frac{\partial y^{1}}{\partial x^{1}}+\frac{\partial y^{2}}{\partial x^{2}}=0
$$

for two unknown functions on an open set $\mathcal{O}$ in $\mathbb{R}^{2}$. Setting $r=2$ we obtain a matrix representation (3) of the form

$$
A=\left(\begin{array}{cc}
\frac{\partial}{\partial x^{1}} & \frac{\partial}{\partial x^{2}} \\
0 & 0
\end{array}\right)
$$

Hence the algebra $\mathcal{A}$ is uniquely determined by the table

$$
\begin{aligned}
& e_{1} e_{1}=e_{1}, \quad e_{1} e_{2}=0 \\
& e_{2} e_{1}=0, \quad e_{2} e_{2}=e_{1},
\end{aligned}
$$

whence

$$
\left(y^{1} e_{1}+y^{2} e_{2}\right)\left(z^{1} e_{1}+z^{2} e_{2}\right)=\left(y^{1} z^{1}+y^{2} z^{2}\right) e_{1} .
$$


One readily sees that the algebra $\mathcal{A}$ is commutative but neither associative nor unital. If

$$
\begin{aligned}
D & =e_{1} \frac{\partial}{\partial x^{1}}+e_{2} \frac{\partial}{\partial x^{2}} \\
y & =y^{1} e_{1}+y^{2} e_{2}
\end{aligned}
$$

then $D y=\left(\frac{\partial y^{1}}{\partial x^{1}}+\frac{\partial y^{2}}{\partial x^{2}}\right) e_{1}$, i.e., the genuine differential equation just amounts to $D y=0$.

\section{The Cauchy-Riemann System}

The last example makes it obvious that the algebra technique is not efficient for arbitrary first order partial differential equations. Indeed, the algebra related to the divergence equation is not associative. Still it is of considerable interest for well motivated equations.

In 1833 W.Hamilton presented a paper for the Irish Academy in which he introduced a formal algebra of real number pairs whose rules of combinations are precisely those for complex numbers $\mathbb{C}$. It is an algebra of rank 2 over $\mathbb{R}$ with basis elements $e, \imath$ and multiplication table

$$
\begin{array}{ll}
e e=e, & e \imath=\imath ; \\
\imath e=\imath, & \imath \imath=-e .
\end{array}
$$

This is an associative commutative division algebra with unity element $e$, i.e.., $\mathbb{C}$ is a field. A function $y: \mathcal{O} \rightarrow \mathbb{C}$ on an open set $\mathcal{O} \subset \mathbb{R}^{2}$ can be written as

$$
y(x)=y^{1}(x) e+y^{2}(x) \imath,
$$

where $y^{1}$ and $y^{2}$ are real-valued functions on $\mathcal{O}$. In this way $y$ can be specified within functions on $\mathcal{O}$ taking their values in $\mathbb{R}^{2}$.

The associated Dirac operator $D$ is the first order partial differential operator

$$
D=e \frac{\partial}{\partial x^{1}}+\imath \frac{\partial}{\partial x^{2}}
$$

whose matrix representation is $D y \mapsto A y$ with

$$
A y=\left(\begin{array}{cc}
\frac{\partial}{\partial x^{1}} & -\frac{\partial}{\partial x^{2}} \\
\frac{\partial}{\partial x^{2}} & \frac{\partial}{\partial x^{1}}
\end{array}\right)\left(\begin{array}{l}
y^{1} \\
y^{2}
\end{array}\right) .
$$

This is the classical Cauchy-Riemann system which plays a crucial role in the function theory of one complex variable.

\section{Quaternions}

Consider the algebra $\mathbb{H}$ of quaternions introduced by W.Hamilton in 1843. It is an algebra of rank 4 over $\mathbb{R}$ with basis elements $e, \imath, \jmath, k$ and multiplication table

$$
\begin{array}{llll}
e e=e, & e \imath=\imath, & e \jmath=\jmath, & e k=k ; \\
\imath e=\imath, & \imath \imath=-e, & \imath \jmath=k, & \imath k=-\jmath ; \\
\jmath e=\jmath, & \jmath=-k, & \jmath \jmath=-e, & \jmath k=\imath ; \\
k e=k, & k \imath=\jmath, & k \jmath=-\imath, & k k=-e .
\end{array}
$$


This is an associative division algebra with unity element $e$. A function $y: \mathcal{O} \rightarrow \mathbb{H}$ on an open set $\mathcal{O} \subset \mathbb{R}^{4}$ can be written as

$$
y(x)=y^{1}(x) e+y^{2}(x) \imath+y^{3}(x) \jmath+y^{4}(x) k,
$$

the components being real-valued functions on $\mathcal{O}$. In this way $y$ can be specified within functions on $\mathcal{O}$ taking their values in $\mathbb{R}^{4}$.

The associated Dirac operator $D$ is the first order partial differential operator

$$
D=e \frac{\partial}{\partial x^{1}}+\imath \frac{\partial}{\partial x^{2}}+\jmath \frac{\partial}{\partial x^{3}}+k \frac{\partial}{\partial x^{4}}
$$

whose matrix representation is $D y \mapsto A y$ with

$$
A y=\left(\begin{array}{cccc}
\frac{\partial}{\partial x^{1}} & -\frac{\partial}{\partial x^{2}} & -\frac{\partial}{\partial x^{3}} & -\frac{\partial}{\partial x^{4}} \\
\frac{\partial}{\partial x^{2}} & \frac{\partial}{\partial x^{1}} & -\frac{\partial}{\partial x^{4}} & \frac{\partial}{\partial x^{3}} \\
\frac{\partial}{\partial x^{3}} & \frac{\partial}{\partial x^{4}} & \frac{\partial}{\partial x^{1}} & -\frac{\partial}{\partial x^{2}} \\
\frac{\partial}{\partial x^{4}} & -\frac{\partial}{\partial x^{3}} & \frac{\partial}{\partial x^{2}} & \frac{\partial}{\partial x^{1}}
\end{array}\right)\left(\begin{array}{l}
y^{1} \\
y^{2} \\
y^{3} \\
y^{4}
\end{array}\right) .
$$

\section{Clifford Algebra}

Elements $\left\{g_{1}, g_{2}, \ldots\right\}$ are said to be generators of an algebra $\mathcal{A}$ if each element $\mathcal{A}$ can be represented as a linear combination with coefficients in $\mathbb{K}$ of products of finitely many elements $g_{j}$.

The Clifford algebra $\mathcal{C}_{n}$ is known to be a unital algebra of rank $r=2^{n}$ over $\mathbb{R}$ with $n$ generators $g_{1}, \ldots, g_{n}$ satisfying $g_{j}^{2}=-e$ for $j=1, \ldots, n$ and $g_{j} g_{k}=-g_{k} g_{j}$ for all $j \neq k$. Clifford algebras are associative.

The first few Clifford algebras are easy to describe, these are $\mathcal{C}_{0}=\mathbb{R}, \mathcal{C}_{1}=\mathbb{C}, \mathcal{C}_{0}=\mathbb{H}$. The 8 -fold periodicity of the Clifford algebras, long known to algebraists, is reminiscent of the 8 -fold periodicity of the stable homotopy groups of the orthogonal group.

Suppose we can find $n$ real matrices $g_{1}, \ldots, g_{n}$ of size $N \times N$ satisfying $g_{j}^{2}=-e$ and $g_{j} g_{k}=$ $-g_{k} g_{j}$ for all $j \neq k$. This corresponds to a real representation of the Clifford algebra $\mathcal{C}_{n}$. The associated Dirac operator is the first order linear differential operator

$$
D=e \frac{\partial}{\partial x^{0}}+g_{1} \frac{\partial}{\partial x^{1}}+\ldots+g_{n} \frac{\partial}{\partial x^{n}},
$$

$e$ being thought of as the $N \times N$ identity matrix.

Such a differential operators on $\mathbb{R}^{n+1}$ has a symbol obtained by replacing $\partial / \partial x_{j}$ by a variable $\sqrt{-1} \xi_{j}$. The Dirac operator $D$ is readily shown to be elliptic, this means its symbol is nonsingular for all $\xi \neq 0$ in $\mathbb{R}^{n+1}$.

\section{Grassmann Algebra}

In 1844 German mathematician Hermann Graßmann published the work "Die Lineale Ausdehnungslehre" in which is formulated a symbolic algebra far surpassing Hamilton's quaternions in generality. Indeed, Graßmann's algebra is nothing less than a full $n$-dimensional vector calculus. 
Once again we start with a vector space $V=\mathbb{K} g_{1}+\ldots+\mathbb{K} g_{n}$. We are interested in defining associative multiplication of vectors satisfying $v v=0$ and $v w=-w v$ for all $v, w \in V$. To this end we first form formal products of basis vectors $g_{1}, \ldots, g_{n}$ in natural order $g^{I}=g_{1}^{i_{1}} \ldots g_{n}^{i_{n}}$, where $I=\left(i_{1}, \ldots, i_{n}\right)$ and each component $i_{1}, \ldots, i_{n}$ takes only two values 0 and 1 . Any power $g_{j}^{0}$ is defined to be $e$, the unity element of $\mathbb{K}$.

We take these $2^{n}$ products as basis elements of some vector space $\Lambda^{\cdot} V$. The elements of $\Lambda^{\cdot} V$ are thus sums

$$
y=y^{0} e+\sum_{j=1}^{n} y^{j} g_{j}+\sum_{1 \leqslant j<k \leqslant n} y^{j, k} g_{j} g_{k}+\ldots+y^{1, \ldots, n} g_{1} \ldots g_{n},
$$

the coefficients being elements of the field $\mathbb{K}$. Multiplication in $\Lambda \cdot V$ is now uniquely defined by two requirements: it should be associative and satisfy $g_{j} g_{k}=-g_{k} g_{j}$ for all $j, k=1, \ldots, n$.

A function $y: \mathcal{O} \rightarrow \Lambda^{\cdot} V$ on an open set $\mathcal{O} \subset \mathbb{R}^{n}$ can be written as

$$
y(x)=\sum_{I} y^{I}(x) g^{I}
$$

where the sum is over all multi-indices $I=\left(i_{1}, \ldots, i_{n}\right)$ with components 0 and 1 and $y^{I}(x)$ are $\mathbb{K}$-valued functions on $\mathcal{O}$. In this way $y$ can be specified within functions on $\mathcal{O}$ taking their values in $\mathbb{K}^{2^{n}}$.

The associated Dirac operator $D$ is the first order partial differential operator

$$
D=g_{1} \frac{\partial}{\partial x^{1}}+\ldots+g_{n} \frac{\partial}{\partial x^{n}}
$$

which is obviously nilpotent, i.e., $D^{2}=0$.

Example 3. For $n=3$, the matrix representation of the Dirac operator $D$ is $D y \mapsto A y$ with

$$
A y=\left(\begin{array}{rrrrrrrr}
0 & 0 & 0 & 0 & 0 & 0 & 0 & 0 \\
\frac{\partial}{\partial x^{1}} & 0 & 0 & 0 & 0 & 0 & 0 & 0 \\
\frac{\partial}{\partial x^{2}} & 0 & 0 & 0 & 0 & 0 & 0 & 0 \\
\frac{\partial}{\partial x^{3}} & 0 & 0 & 0 & 0 & 0 & 0 & 0 \\
0 & -\frac{\partial}{\partial x^{2}} & \frac{\partial}{\partial x^{1}} & 0 & 0 & 0 & 0 & 0 \\
0 & -\frac{\partial}{\partial x^{3}} & 0 & \frac{\partial}{\partial x^{1}} & 0 & 0 & 0 & 0 \\
0 & 0 & -\frac{\partial}{\partial x^{3}} & \frac{\partial}{\partial x^{2}} & 0 & 0 & 0 & 0 \\
0 & 0 & 0 & 0 & \frac{\partial}{\partial x^{3}} & -\frac{\partial}{\partial x^{2}} & \frac{\partial}{\partial x^{1}} & 0
\end{array}\right)\left(\begin{array}{l}
y^{0} \\
y^{1} \\
y^{2} \\
y^{3} \\
y^{4} \\
y^{5} \\
y^{6} \\
y^{7}
\end{array}\right)
$$

For $q=0,1, \ldots, 3$, we denote by $\Lambda^{q} V$ the subspace of $\Lambda^{\cdot} V$ spanned by the $q$-fold products $g^{I}$, i.e., those with $|I|=q$. The basis of $\Lambda^{q} V$ consists of all $g^{I}$ with $|I|=q$ put in the lexicographic order. Then $\Lambda^{\cdot} V$ splits into the direct sum $\Lambda^{0} V \oplus \ldots \oplus \Lambda^{3} V$ which implies

$$
A y=0 \oplus \nabla y^{0} \oplus \operatorname{rot}\left(\begin{array}{c}
y^{1} \\
y^{2} \\
y^{3}
\end{array}\right) \oplus\left(\begin{array}{ccc}
\frac{\partial}{\partial x^{3}} & -\frac{\partial}{\partial x^{2}} & \frac{\partial}{\partial x^{1}}
\end{array}\right)\left(\begin{array}{c}
y^{4} \\
y^{5} \\
y^{6}
\end{array}\right)
$$

rot being the rotation operator. 


\section{Cross Product}

If the dimension $n$ of $V$ is odd, then the dimensions of $\Lambda^{q} V$ and $\Lambda^{q+1} V$ coincide provided

$$
q=\frac{n-1}{2} .
$$

We are therefore led to an algebra structure on $\mathbb{K}^{\left(\begin{array}{c}n \\ q\end{array}\right)}$ related to the differential operator $D$ of the previous section acting on functions taking their values in the space $\Lambda^{q} V$.

For $n=3$ this just corresponds to the usual cross product on $\mathbb{K}^{3}$. In fact, the operator in question is

$$
A y=\left(\begin{array}{rrr}
-\frac{\partial}{\partial x^{2}} & \frac{\partial}{\partial x^{1}} & 0 \\
-\frac{\partial}{\partial x^{3}} & 0 & \frac{\partial}{\partial x^{1}} \\
0 & -\frac{\partial}{\partial x^{3}} & \frac{\partial}{\partial x^{2}}
\end{array}\right)\left(\begin{array}{l}
y^{1} \\
y^{2} \\
y^{3}
\end{array}\right),
$$

which is due to Example 3. The related algebra is determined by the multiplication table

$$
\begin{array}{lll}
e_{1} e_{1}=0, & e_{1} e_{2}=e_{1}, & e_{1} e_{3}=e_{2} ; \\
e_{2} e_{1}=-e_{1}, & e_{2} e_{2}=0, & e_{2} e_{3}=e_{3} ; \\
e_{3} e_{1}=-e_{2}, & e_{3} e_{2}=-e_{3}, & e_{3} e_{3}=0 .
\end{array}
$$

It is easy to see that this algebra is neither commutative nor associative while the cross product in $\mathbb{K}^{3}$ is known to be of considerable importance both in physics and geometry.

\section{Lie Algebras}

While Cayley's algebra of octonions was the first nonassociative algebra to be discovered, it is really not much more than a mathematical curiosity. The most significant type of nonassociative algebra, now known as a Lie algebra, was introduced in 1876 by the Norwegian mathematician Sophus Lie. This algebras were the distillation of Lie's study of the structure of groups of spatial transformations, which in turn involved the idea of an infinitesimal transformation in space, that is, one which moves any point in space an infinitesimal distance. Infinitesimal transformations may be added and multiplied by real numbers in a natural way. Given two infinitesimal transformations $X$ and $Y$, we may also consider their composite $X Y$, that is, the transformation that results by applying first $Y$ and then $X$. Now $X Y$ and $Y X$ are in general not the same, nor indeed are they infinitesimal transformations. However, the transformation $X Y-Y X$ is infinitesimal. It is called the commutator or Lie product of $X$ and $Y$ and is written $[X, Y]$. If we regard this Lie product as a multiplication operator on infinitesimal transformations, then these form a nonassociative algebra over $\mathbb{R}$. Moreover, the Lie product satisfies the laws

1) $[X, X]=0$;

2) $[X, Y]=-[Y, X]$

3) $[[X, Y], Z]+[[Y, Z], X]+[[Z, X], Y]=0$.

Equation 3) is known as the (C. J. G.) Jacobi identity. An algebra satisfying 1), 2) and 3 ) is called a Lie algebra. A noteworthy example of a Lie algebra is the algebra of vectors in 3 -dimensional space with multiplication given by Graßmann's vector product.

Lie algebras of point symmetries (shortly symmetry algebras) have become a crucial tool for classification of differential equations and construction of explicit solutions, cf. [1]. 


\section{References}

[1] P.J.Olver, Equivalence, Invariants, and Symmetry, Cambridge University Press, 1995. 